\title{
A Performance Analysis Tool For Performance-Driven Micro-Cell Generation.
}

\author{
R.Peset LLopis, R.J.H.Koopman, H.G. Kerkhoff and J.A.Braat. \\ IC-Technology and Electronics Group. \\ University of Twente, P.O.Box 217, 7500 AE Enschede, The Netherlands.
}

\begin{abstract}
A new method is presented to determine the power dissipation and propagation-delay time of small logical blocks (micro-cells). This method is a combination of the RC-tree and the macro modeling methods. It is a fast and accurate method, three orders of magnitude faster that SPICE, while the maximal error is 10 percent. This method will be used in a performance-driven micro-cell generator for a sea-of-gates environment.
\end{abstract}

\section{Introduction}

With the growing integration of todays and future electronic circuits and the increasing possibilities of technology, it is getting difficult to meet the specifications of the entire circuit. These specifications do not only require a logical description, but also demands with regard to constraints. Among these are operating speed, noise margins, power dissipation and area. This paper presents a layout-generation technique for a sea-of-gates environment that takes these specifications into account. It aims to use all the possibilities offered by todays technology in order to improve performance.

A sea-of-gates array consists of an array of core-cells [1]. A core-cell is the smallest repetitive element of this array, and consists of $\mathrm{n}$ - and p-transistors. A sea-of-gates environment is a semi-custom technique, which is based on a transistor array equal for all applications, and two or three metal layers that are different for each application. Figure la shows a part of a transistor array [1]. A circuit is realized by generating the interconnect layers. Figure 1 b shows an implementation of an eight-input NAND. The main difference with a gate-array is the absence of separate interconnection areas. It offers therefore the advantages of semi-custom gate-arrays, such as quick turn-around times and low cost without its disadvantages, as low densities and medium performance. A micro-cell is defined as a small logical block, the most complex one being a full adder. It is realized in a sea-of-gates array and consists therefore of at least one core-cell with interconnect.

An elegant way to meet the performance specifications is to use performance-driven circuit-generation techniques. A macro-assembler, employing logic synthesis techniques is used to decompose the global specifications into micro-cells. This decomposition must take the parasitic capacitances and resistances caused by the interconnect into account, since they influence the performance. From this, specifications for micro-cells can be deduced. These micro-cells are generated by a performance-driven micro-cell compiler (PMCC). The PMCC must also take the interconnections that cross the generated micro-cell into account.

In this paper, the development of a fast and accurate performance-analysis tool is discussed, which is part of the performance-driven micro-cell compiler.

\section{Performance criteria}

A basic criterion is which performance constraints can be of interest in a PMCC environment. It is obvious that the maximum operational frequency, the total power dissipation and the total area of an entire chip are very important aspects in chip design. The logic synthesis techniques must translate these global constraints to micro-cell constraints, resulting in a propagation delay, a power dissipation and an area and shape at micro-cell level. Improving the operational frequency increases the danger of noise susceptibility. Noise margins are often compromised to improve speed. Therefore it is very important to introduce a lower bound on noise margins to avoid a high noise susceptibility. Therefore the performance constraints for the PMCC are chosen to be:

- Maximum propagation delay.

- Maximum power dissipation.

- Maximum area.

- Minimum noise margins.

The PMCC must be able to predict the performance of the generated micro-cells to verify if these constraints have been met.

The next point of interest is the tradeoff between accuracy and CPU-time. Since meeting the constraints can be seen as an optimization technique, an iterative approach is used for the PMCC; small changes, e.g. adding transistors in parallel or in series, or adding buffers, are implemented in the micro-cell until the constraints are met. This implies very fast iterations in order to prevent large CPU-times. The accuracy 
of the PMCC must be comparable to that of the logic synthesis technique, resulting in a required accuracy of 10 percent.

3. Current performance prediction techniques

The three most important performance prediction methods in literature, will be briefly discussed next.

The first method consists of circuit simulation techniques (SPICE and [2]). This method calculates analog waveforms and offers therefore high accuracies, but suffers from long CPU-times.

The second method is very often used in applications with fixed layout libraries, such as gate-arrays. It consists of macro modeling techniques [3]. The shape of the output waveform of each micro-cell (delay-time and rise/fall time) is determined as a function of several parameters, usually the load capacitance and the input transition time. This method offers high accuracies (under 5 percent) and small computational times. Using macro modeling techniques in a performance-driven layout generator results in a tremendous increase in the total number of parameters that determine the shape of the output waveform. This is because the layout is no longer fixed by a library. The layout parameters, such as capacitances and resistances of the interconnect from each micro-cell, must also be taken into account. Macro modeling is therefore not practical in our application.

The third method is very often used in performance-driven applications, such as full-custom transistor sizing algorithms. These applications use RC-tree calculations [4-5] to approximate each transistor by a linear RC network (fig. 2a-2b). Several models and calculation techniques with a variation of accuracies can be found [5]. The best methods offer an average error per micro-cell of 20 percent with regard to delay calculations. This method offers a high computing speed, but can not be used to predict accurate power dissipation values.

Table 1 shows the advantages and disadvantages of these three methods, together with the requirements of the PMCC. The last column represents the practicability of these methods. Macro modeling is not practical for the PMCC, due to the large total number of equations.

\begin{tabular}{|c|c|c|c|}
\hline Method & CPU-time & Accuracy & Practicability \\
\hline Required & + & + & + \\
\hline Circuit simulation & - & + & + \\
\hline Macro modeling & + & + & - \\
\hline RC-tree method & + & - & + \\
\hline
\end{tabular}

4. A new performance prediction technique The new technique is a combination of the RC-tree and the macro modeling methods. It aims to combine the CPU-efficiency of the RC-tree method with the high accuracy of the macro modeling method. An increase in accuracy is achieved by using macro modeling and substituting only the non-switching transistors by RC networks. The last has two consequences:

- First, the most important transistors (the switching ones) are not substituted by RC networks, resulting in an increase in delay accuracy.

- Second, it is also possible to take the short-circuit power dissipation into account, resulting in an increase in power accuracy. The dissipated power in the case of full-CMOS, is given by:

$P C M O S=P_{\text {static }}+P_{\text {dymamic }} \approx P_{\text {dynamic }}$

$$
=P_{\text {short-circuit }}+P_{\text {capacitor }}
$$

$P_{\text {static }}$ is the static power dissipation and is caused by the reverse bias-leakage current between diffusion regions and the substrate. Pdynamic is the power dissipation during a transient on the input. The static dissipation is much smaller than the dynamic dissipation and can be neglected in most CMOS applications. Pshort-circuit is the short-circuit power dissipation and occurs during a transient on the input, when there is a path of conducting $\mathrm{n}$ - and conducting $\mathrm{p}$-transistors $\left(V_{T n}<V_{\text {in }}<V_{d d}-V_{T p}\right)$. $V_{T n}$ and $V_{T p}$ denote the threshold voltage of the $n$ - and p-transistor respectively, $V_{\text {in }}$ the input voltage and $V_{d d}$ the power supply-voltage. $P_{\text {capacitor }}$ is caused by charging and discharging the capacitors. The short-circuit power dissipation can be larger than the capacitor power dissipation [6] and must be taken into account. Substitution of the switching transistors by RC networks, as done in RC-tree calculations, will not result in a short-circuit path and leads to an underestimation of the power dissipation.

\section{The basic structures}

The main goal of the new performance prediction method is to reduce all switching micro-cells to a few basic structures. The choice of the basic structures is of great importance for this method. The total number of structures depends on the required accuracy and the used design style. Most current gate-array libraries use the full-CMOS design style with in addition transmission gates. They can be dealt with only two basic structures.

The first basic structure is used when the switching transistors are located in the full-CMOS part. An inverter with complex RC networks (fig. 3b) is the result of substituting the non-switching transistors by $\mathrm{RC}$ networks. Transmission gates and the load capacitance at the output of this inverter are modeled by RC network RC3.

The second basic structure is used when a transmission gate contains the switching transistors. This structure consists of a transmission gate with RC networks (fig. 3a). The non-switching full-CMOS micro-cell at the input of the transmission gate is modeled by RC network RC1. 
Transmission gates and the load capacitance at the output are modeled by RC network RC2.

The same basic structures are used to predict the propagation delay and power dissipation. The only differences are the $R$ and $C$ values of the $R C$ networks, which are used to replace the non-switching transistors. The exact $R$ and $C$ values are determined by accurate SPICE simulations.

Accurate SPICE simulations are also used to determine relations between the performance of these basic structures and their resistances, capacitances, transistor widths, transistor lengths and the input rise/fall time $T_{\mathrm{rf}}$. These relations are stored in a table. Table lookup operations instead of SPICE simulations are carried out during a performance prediction session.

If this reduction is possible without losing performance data and the performance of these basic structures can be determined using a lookup table, then one can determine the performance of all reducible micro-cells.

\section{The algorithm}

The performance prediction method can be divided into four different phases:

1) Reducing a micro-cell into stages.

2) Replacing the non-switching transistors by linear RC networks.

3) Reducing the complex RC networks to compact RC networks.

4) Determining the performance parameters.

These phases will be explained in detail in the following.

\section{Phase 1}

The new performance prediction technique can only be applied to single stages. A stage is defined as a part of a circuit that has a single $n$-transistor and a single p-transistor ( $\mathrm{m}_{1}$ and $\mathrm{m} 2$ ) as input, and no transistor gates in the data path of the stage (fig. 4a). This is not a severe limitation, since most micro-cells consist of one stage, and more complex micro-cells (i.e. full adder) can easily be divided in several stages. Subsequently, each of these stages can be treated separately.

\section{Phase 2}

Assume, as most frequently occurs, that there is only a single switching $\mathrm{n}$ - and a single switching $\mathrm{p}$-transistor per stage ( $\mathrm{m}_{1}$ and $\mathrm{m}_{2}$ ) and that this switching pattern results in a transient at the output. Other cases are not of interest. Replacing the non-switching transistors of figure $4 \mathrm{a}$ by $\mathrm{RC}$ networks results in an inverter with complex RC networks at the sources and drains of the $n$ - and p-transistors (fig. 4b). This replacement is shown for transistor $\mathrm{m} 3$.

Some RC-tree applications [5] use RC networks that are a function of the input and output rise/fall times. Simulations showed that the effects of these rise/fall times can also be modeled by two additional capacitors. Therefore fixed RC networks with two additional capacitors ( $C_{1}$ and $C_{2}$ of fig. $4 c$ ) are used to model these effects.

Parasitic capacitances and resistances of the interconnect can be easily included in these RC networks.

\section{Phase 3}

The complex RC networks of the inverter (fig. 4c) are simplified, using an Elmore-based method [7-9], to obtain simplified RC networks (fig. 4d). Finally, the capacitors connected to the power-supply can be omitted while others can be replaced by a single capacitor, resulting in a simplified network (fig. 4e). This network has sufficient properties from the original network to be used for accurate performance evaluation.

\section{Phase 4}

The reduction applied in the previous phase results in two basic structures, one for power and one for delay predictions. Each contains thirteen parameters (four resistances $R_{1} . . R_{4}$, five capacitors $C_{1} . . C_{5}$ and four transistor parameters $W_{n}, L_{n}$, $W_{p}$ and $L_{p}$ ), depicted in fig. $4 \mathrm{e}$. The relations stored in a lookup table, discussed in paragraph 5 , are used to determine the performance as function of these parameters and the input rise/fall time $\mathbf{T}_{\mathbf{r f}}$.

\section{Results}

This method has been implemented in a C-program. This program does not yet use sophisticated programming techniques to improve speed. The lookup table has been generated using SPICE simulations. Five CPU-hours were needed to generate this table, having a size of $7 \mathrm{KBytes}$. The exact $R$ and $C$ values of the RC networks, which are used to replace the non-switching transistors, have also been determined by SPICE simulations.

Different test circuits have been used to determine the accuracy and time consumption of the new program in comparison to a SPICE simulation. The input of the new program consists of a SPICE-description of a test circuit. Each stage of this circuit is reduced to a basic structure. The power dissipation and propagation delay values of each stage are determined using table lookup operations.

SPICE simulations using the same input file are carried out to determine the same performance parameters.

Figure $6 \mathrm{a}-6 \mathrm{~b}$ show the propagation delay and the power dissipation of a four input NOR, as function of the input rise/fall time and the load capacitance. The curves represent the SPICE values. These figures show a maximal error of 10 percent.

Different test structures, such as NANDs, NORs, full adders, show an overall accuracy of 10 percent. The simulated (SPICE) and the predicted (PMCC) performance parameters are shown in figure $6 \mathrm{c}-6 \mathrm{~d}$, together with the +10 and -10 percent limits. These figures demonstrate, that the maximal 
prediction error is 10 percent. The CPU-times for an Apollo DN10000 to determine the performance of a full adder (fig. 5 ) are shown in table 2 . The new program is able to determine the performance of a full adder (the most complex micro-cell) more than three orders of magnitude faster than SPICE. The output waveforms of the same full adder are shown in figure 7. The PMCC waveform is the dotted one.

\begin{tabular}{|c|c|c|}
\hline & SPICE & PMCC \\
\hline CPU-time & $40 \mathrm{~s}$ & $20 \mathrm{~ms}$ \\
\hline
\end{tabular}

\section{Conclusions and future work}

A new performance determination method has been presented, to be used in a performance-driven micro-cell compiler for a sea-of-gates environment. A C-program has been written that is able to predict the power dissipation and the propagation delay of full CMOS micro-cells. This program uses a lookup table having a size of 7 KBytes, which was generated in five CPU-hours.

A full adder is predicted more than 3 orders faster in comparison to SPICE. The maximal prediction error is 10 percent, in contrast to an average error of 20 percent for the RC-tree method

This method will be extended to determine also the noise margins and to make it applicable for other design styles.

\section{Acknowledgements}

The authors wish to thank their colleagues at the Philips Research Laboratories Eindhoven and at the Technical Universities of Delft and Eindhoven for a stimulating cooperation. This work is supported by the Dutch Innovative Research Projects program part VLSI-design (IC-TEL 88.038).

\section{References}

[1] R.J.H. Koopman, R. Peset LLopis and H.G. Kerkhoff, "Digital CMOS Sea-of-Gates Core Cells and Master Images", Proc. 16th European Solid-State Circuit Conf. (ESSCIRC) Grenoble, France, September 1990, pp 245-248.

[2] P. Subramaniam, "Table Models for Timing Simulation", Custom Integrated Circuits Conf. 1984, pp 310-314.

[3] L.M. Brocco, S.P. McCormick and J. Allen, "Macromodeling CMOS Circuits for Timing Simulation", IEEE Trans. on Computer-Aided Design, Vol. 7, No. 12, December 1988, pp 1237-1249.

[4] F.W. Obermeier and R.H. Katz, "An Electrical Optimizer that considers Physical Layout", IEEE Design Automation Conf. 1988, pp 453-459.

[5] J.K. Ousterhout, "A Switch-Level Timing Verifier for Digital MOS VLSI", IEEE Trans. on Computer-Aided Design, Vol. CAD-4, NO. 3, July 1985, pp 336-349.
[6] H.J.M. Veendrick, "Short-Circuit Dissipation of Static CMOS Circuitry and its Impact on the Design of Buffer Circuits", IEEE J. of Solid-State Circuits, Vol. SC-19, No. 4, August 1984, pp 468-473.

[7] P. Penfield and J. Rubinstein, "Signal Delay in RC Tree Networks", 18th Design Automation Conference, 1981, pp 613-617.

[8] S.L. Su, V.B. Rao and T.N. Trick, "A Simple and Accurate Node Reduction Technique for Interconnect Modeling in Circuit Extraction", Int. Conf. on Computer-Aided Design, 1986, pp 270-273.

[9] J.L. Wyatt, "Signal Delay in RC Mesh Networks", IEEE Trans. on Circuits and Systems, Vol. CAS-32, No 5, May 1985, pp 507-509.

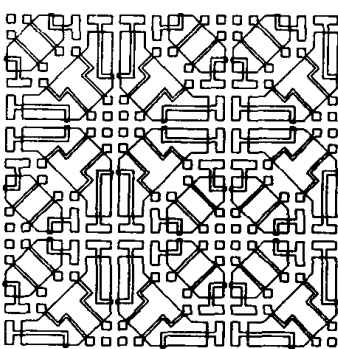

Fig. 1a A sea-of-gates array.

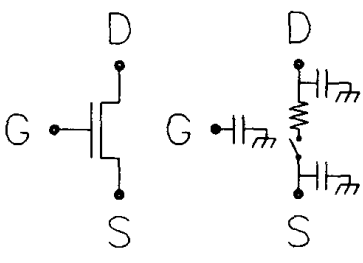

Fig. $2 a$

Fig. 2b

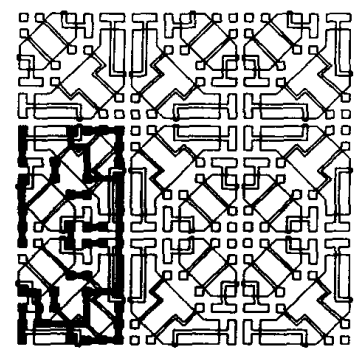

Fig. 16 A realization of an eight input NAND.

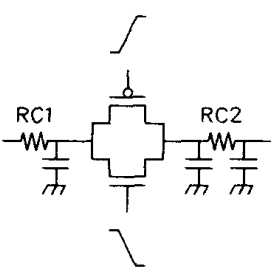

A MOS transistor. Its $\mathrm{RC}$ replacement.

Fig. 3a The transmission gate basic structure.

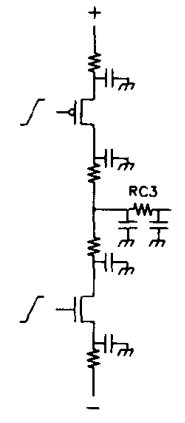

Fig. 3b The inverter basic structure.

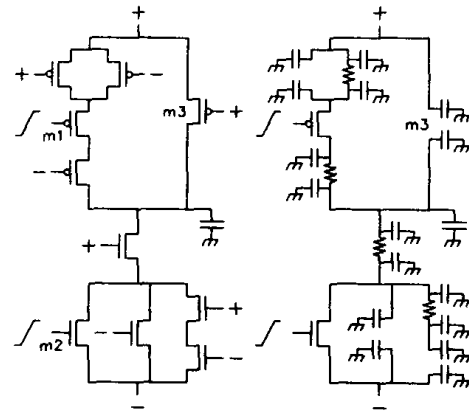

Fig. 4a The original Fig. $4 \mathrm{~b}$ After transistor micro-cell. 


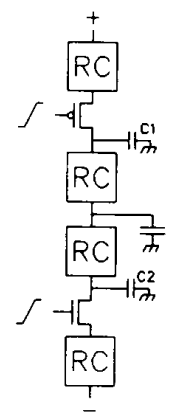

Fig. 4c After adding $\mathrm{C} 1$ and $\mathrm{C} 2$.

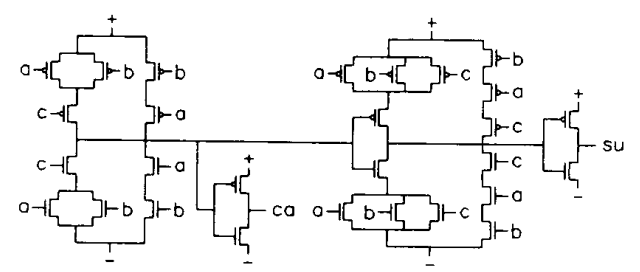

Fig. 5 The used full adder.

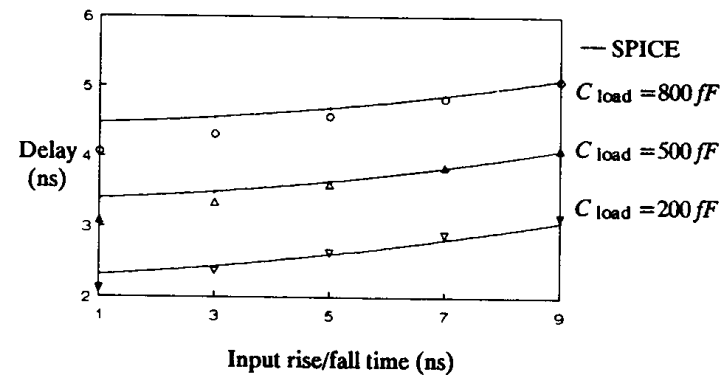

Fig. 6a The propagation delay of a four input NOR.

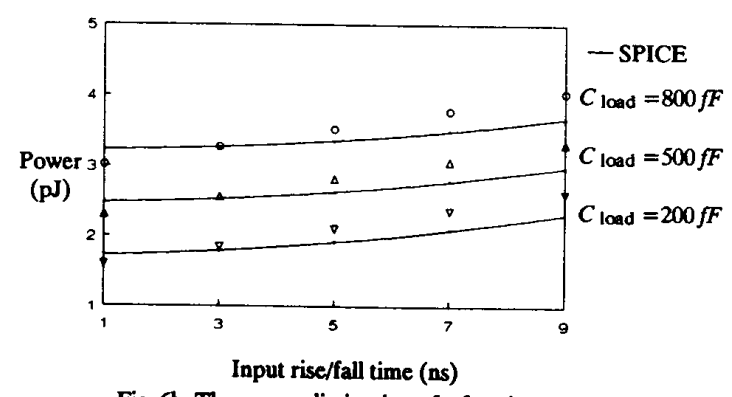

Fig. $6 \mathrm{~b}$ The power dissipation of a four input NOR

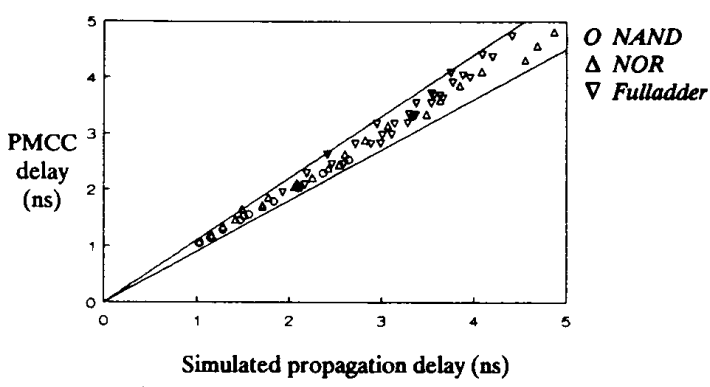

Fig. $6 c$ The overall propagation delay accuracy.

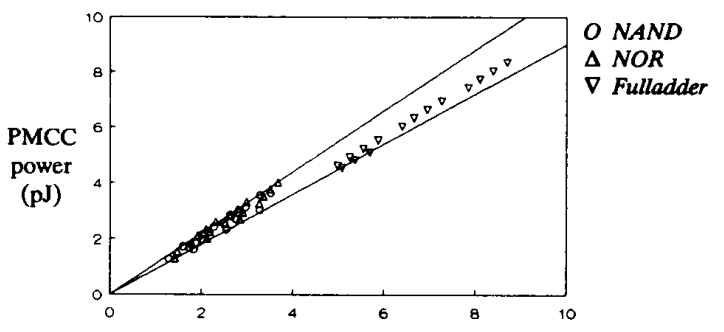

Simulated power dissipation $(\mathrm{p})$

Fig. 6d The overall power dissipation accuracy.

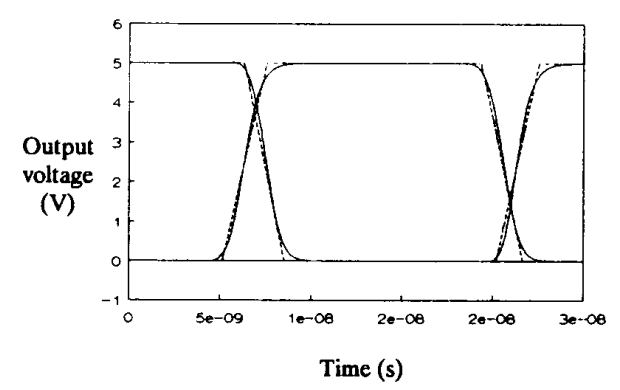

Fig. 7 SPICE and PMCC full adder output waveforms. 